\title{
An overview of STRUCTURE: applications, parameter settings, and supporting software
}

\author{
Liliana Porras-Hurtado ${ }^{1,2+}$, Yarimar Ruiz ${ }^{2+}$, Carla Santos ${ }^{2+}$, Christopher Phillips ${ }^{2 *}$, Ángel Carracedo ${ }^{2,3}$ \\ and Maria V. Lareu ${ }^{2}$ \\ Universidad Tecnológica de Pereira, Pereira, Colombia \\ ${ }^{2}$ Forensic Genetics Unit, Institute of Legal Medicine, University of Santiago de Compostela, Santiago de Compostela, Spain \\ ${ }^{3}$ Genomics Medicine Group, CIBERER, University of Santiago de Compostela, Santiago de Compostela, Spain
}

\section{Edited by:}

Xiaofeng Zhu, Case Western

Reserve University, USA

\section{Reviewed by:}

Guimin Gao, Virginia

Commonwealth University, USA

Qunyuan Zhang, Washington

University School of Medicine, USA

Huaizhen Qin, Tulane University,

USA

\section{*Correspondence:}

Christopher Phillips, Forensic

Genetics Unit, Institute of Legal

Medicine, University of Santiago de

Compostela, Rúa San Francisco s/n,

15782 Santiago de Compostela,

Galicia, Spain

e-mail: c.phillips@mac.com

${ }^{\dagger}$ These authors have contributed equally to this work.
Objectives: We present an up-to-date review of STRUCTURE software: one of the most widely used population analysis tools that allows researchers to assess patterns of genetic structure in a set of samples. STRUCTURE can identify subsets of the whole sample by detecting allele frequency differences within the data and can assign individuals to those sub-populations based on analysis of likelihoods. The review covers STRUCTURE's most commonly used ancestry and frequency models, plus an overview of the main applications of the software in human genetics including case-control association studies (CCAS), population genetics, and forensic analysis. The review is accompanied by supplementary material providing a step-by-step guide to running STRUCTURE.

Methods: With reference to a worked example, we explore the effects of changing the principal analysis parameters on STRUCTURE results when analyzing a uniform set of human genetic data. Use of the supporting software: CLUMPP and distruct is detailed and we provide an overview and worked example of STRAT software, applicable to CCAS.

Conclusion: The guide offers a simplified view of how STRUCTURE, CLUMPP, distruct, and STRAT can be applied to provide researchers with an informed choice of parameter settings and supporting software when analyzing their own genetic data.

Keywords: STRUCTURE, CLUMPP, distruct, STRAT, population structure, case-control association studies, stratification

\section{AN OVERVIEW OF THE STRUCTURE PROGRAM}

STRUCTURE is a freely available program for population analysis developed by Pritchard et al. (2000a). STRUCTURE analyses differences in the distribution of genetic variants amongst populations with a Bayesian iterative algorithm by placing samples into groups whose members share similar patterns of variation. STRUCTURE both identifies populations from the data and assigns individuals to that population representing the best fit for the variation patterns found. Typically STRUCTURE is the first step in examining population structures that emerge from the sample set to provide a preamble to further genetic analysis or to infer the origins of individuals with unknown population characteristics, especially when population admixture has occurred. As STRUCTURE uses the core Bayesian principle of comparing likelihoods, prior information about study samples can be supplied to further shape the analysis. For example, information about sampling location can be input-a characteristic, if shared between individuals that can be associated with their genetic proximity. The definition of populations can be assessed from geographical distribution, but is also often based on alternative criteria, including the phenotype, behavior, and ecology of sampled individuals, while linguistic and cultural characteristics can also define human populations. Therefore, it is important to assess whether assignment of individuals to populations with non-genetic criteria is consistent with genetic patterns detected between populations (Pritchard et al., 2000a; Jobling et al., 2004; Waples and Gaggiotti, 2006).

STRUCTURE uses a systematic Bayesian clustering approach applying Markov Chain Monte Carlo (MCMC) estimation. The MCMC process begins by randomly assigning individuals to a pre-determined number of groups, then variant frequencies are estimated in each group and individuals re-assigned based on those frequency estimates. This is repeated many times, typically comprising 100,000 iterations, in the burnin process that results in a progressive convergence toward reliable allele frequency estimates in each population and membership probabilities of individuals to a population.

Measurement of the assumed number of populations uses the MCMC estimation and is performed separately from the burnin. STRUCTURE performs individual analyses for each assumed population number from one to a reasonably appropriate number for the sampling regime. STRUCTURE applies a model to the data of $K$ assumed populations or genetic groups, each characterized by a subset of allele frequencies identified in the data. Commonly $K$ is not readily defined by the user for the sample set, although this parameter must be pre-selected. Therefore, an appropriate first step is to calculate the likelihood of the data for a range of $K$ values by creating posterior probabilities of $K$, termed $X$ and 
written: $X \mid K$. Since $K$ is not an absolute value, user-defined values should be considered carefully, taking into account characteristics of the sampled populations. Running a range of prescribed $K$ settings to obtain their $X$ values normally creates probabilities smaller than those for the most appropriate $K$ value but beyond this probabilities tend to be very similar for higher $K$ values. Therefore, plots of $X$ values typically progress to a plateau for levels of $K$ beyond the most applicable number of detected populations, so the smallest stable $K$ value represents the optimum value. Kalinowski (2011) notes that better clusters are created applying the most realistic $K$ values, so it is prudent to obtain the smallest value of $K$ that maximizes the global likelihood of dataan approach capturing the major underlying population structure in the data without overestimating it.

During each analysis membership coefficients summing to one are assigned to individuals for each group. The membership coefficient matrix, termed the individual $Q$-matrix, is generated with rows for the number of individuals analysed and columns for $K$ clusters. The average individual membership coefficients to each population form the population Q-matrix. If admixture is not a factor for the population samples analysed, posterior probabilities of belonging to each of $K$ groups are calculated for each individual and a sample can be considered a member of the group with the highest probability. If admixture is considered membership coefficients are made across multiple clusters.

Bayesian population analysis methods equate allele frequencies that define the population and the frequencies found in individuals identified as originating from that population. Therefore, the ability of Bayesian methods to differentiate populations amongst a set of samples is severely restricted from limited sample sizes and small marker numbers (Corander et al., 2003; Corander and Marttinen, 2006). Genetic markers applied to STRUCTURE analyses ideally show selective neutrality, low mutation rates and absence of linkage disequilibrium (LD) (Pritchard et al., 2000a; Corander et al., 2003). In short, they are treated as independent variables and so-called naïve Bayesian approaches assume independence, but without a guarantee this applies. However, enhancements since STRUCTURE 2.3.1 permit inclusion of weakly linked markers with some degree of non-independence (Falush et al., 2003). Additionally, Falush et al. (2007) describe an algorithm that allows application of all available models to dominant markers (loci often characterized by genotype ambiguity).

Note that SNPs (single nucleotide polymorphisms), as binary markers, have lower variability than multiple-allele loci, requiring much smaller sample sizes to obtain accurate allele frequency estimates. Shi et al. (2010) suggested population samples as low as four individuals are sufficient to provide reliable data by demonstrating subsamples of four taken from much larger population samples give very similar posterior median population parameters. Microsatellites require considerably larger samples sizes than SNPs to reliably capture patterns of variability in a population. Lastly, recent forensic guidelines for microsatellite population surveys recommend genotyping a minimum 500 samples (Carracedo et al., 2013).

\section{ANCESTRY AND ALLELE FREOUENCY MODELS}

Two terms are relevant to a review of the estimation of ancestry: local ancestry and global ancestry. Local ancestry estimates the extent to which each person's genome is divided into chromosome segments of definite ancestral origin. Global ancestry estimates the proportion of ancestry from each contributing population, considered as an average over the individual's entire genome (Alexander et al., 2009). STRUCTURE only estimates global ancestry by implementing different models of population structure to the data. Selection of the most appropriate model depends on the user's data and study objectives. Two ancestry models applied by STRUCTURE are the no admixture and admixture models. If there is no prior knowledge about the origin of the populations under study or if there is reason to consider each population as completely discrete, the no admixture model is appropriate. However, admixture between populations is a common characteristic such that a large proportion of sampled individuals can have recent ancestors from multiple populations. In these cases knowing the approximate median value of the ancestral population proportions for each individual and/or their populations of origin is a very useful part of the characterization of the populations under study. In these cases the admixture model is more appropriate. Both models can be used with consideration for sampling location information by applying the prior model parameter: LOCPRIOR to the population model (Hubisz et al., 2009). This option can be used when there is additional sample-characteristic data available to the user, including: linguistic, geographical, cultural, or phenotypic information. The LOCPRIOR parameter is particularly informative when there are weak population structure signals-a situation that can result from using reduced numbers of markers, small sample sizes or due to close relationships between populations.

The third model parameter is linkage, based on the admixture model and this is designed to deal with admixture LD: the characteristic of extended LD found in admixed populations and often deliberately sought in association studies. This model was outlined by Falush et al. (2003) and provides more accurate estimates of statistical uncertainty when linked markers are used.

No admixture, admixture, and linkage models can also be analysed as part of the USEPOPINFO model. This model uses the population labels to calculate the probability that each individual has of originating from the assumed populationindividuals with low probabilities can be considered as hybrids or migrants (Pritchard et al., 2000a). This parameter should be used cautiously-applied only when the population labels are well defined beforehand and correspond almost exactly to the groups ultimately defined by the STRUCTURE results. The disadvantage of the USEPOPINFO model arises with the posterior handling of the results. The individual Q-matrix comprises probabilities (and not ancestry membership proportions) that are presented in a format that is incompatible with post-hoc data-processing software such as CLUMPP or distruct.

All the models considered until now can be used in conjunction with an alternative approach to USEPOPINFO - the POPFLAG model. POPFLAG considers the specified information about the population of origin of a portion of the individuals to help infer the ancestry of other samples with unknown origin. This option should also be used with caution because selected samples will be treated as the "reference" set (preassigned POPFLAG $=1$ ) so allele frequency estimates are based 
on a reduced sub-set of samples and will directly affect the grouping of the unknown individuals (pre-assigned POPFLAG $=0$ ). POPFLAG is an artificial model that assesses the individual probability of being part of a particular population, but it can be useful if the objective is to efficiently group individuals/populations by comparison with a particularly well-defined and studied reference data set (Pritchard et al., 2000a). One such reference set, widely applied to human population genetics studies, is the $\mathrm{CEPH}$ human genome diversity panel (HGDP-CEPH) (Cann et al., 2002) with the advantage that population structure has been identified in this sample set in a wide range of studies using different markers and a variety of data depths, but with consistent findings (Rosenberg et al., 2002; Enoch et al., 2006; Abdulla et al., 2009). When the POPFLAG model is used in conjunction with the USEPOPINFO model the individual Q-matrix is composed of two distinct parts: for POPFLAG $=1$ individual's the matrix presents probabilities, while for POPFLAG $=0$ individuals ancestry membership proportions are given according to the admixture model defined (no admixture, admixture, or linkage).

Related subjects should be detected then excluded from reference data since shared variation is inflated in frequency causing estimation bias that consequently affects analysis of the ancestry of study samples. Prudent checks of reference data are clearly advisable, for example, an audit of the HGDP-CEPH samples was published by Rosenberg (2006) where two atypical individuals, 13 duplicates, and as many as 77 first-degree relative pairs were identified and removed. Related subjects can regularly form part of the study set and this approach is central to linkage studies in genetic epidemiology as these seek loci with correlations between traits of interest and patterns of transmission of DNA sequence over generations in a known pedigree (Astle and Balding, 2009).

Two allele frequency models are available. The correlated allele frequencies model assumes a level of non-independence, so is more conservative. The independent allele frequencies model requires knowledge about the correlation levels across populations-allele frequencies should be reasonably different in distinct populations. The correlated allele frequencies model provides greater power to detect distinct populations that are particularly closely related, although this model will give the same results as the independent allele frequencies model in the absence of high levels of correlation across populations. Therefore, it is prudent to use the correlated allele frequencies model since this will guarantee that a previously undetected correlation is identified, but without affecting the results if no such correlation exists.

Summarizing the range of models that can be applied to a uniform set of genetic data, Figure 1 shows bar plots produced by STRUCTURE when applying each of the models described above. The application of each model is outlined in detail in the Supplementary Material 1, section 5.

It is noteworthy that a level of finesse exists when implementing analysis models in STRUCTURE. All the models described above include specific statistical parameters that can be adjusted to more sensitive values, including $r$ (informativeness of the sampling location data), alpha (relative admixture levels between populations), and lambda (quantifies the independence between markers in terms of their allelic frequency distribution).

\section{ASSIGNMENT OF INDIVIDUALS TO A POPULATION AND CHOICE OF MARKERS}

Assigning individuals to populations is often useful in population genetics studies (Pritchard et al., 2000a) where making a population classification can provide an inference of individual ancestry that may not have been adequately defined beforehand (Royal et al., 2010). The typical approach has already been described for STRUCTURE: establishing pre-defined populations from reference samples and assigning individuals of unknown origin to these populations. Reference samples provide allele frequency estimates in each population that are then used to compute the likelihood of membership of samples of unknown origin to any population (Pritchard et al., 2000a).

When using small numbers of markers, highly differentiated genetic variants are more informative per locus than randomly chosen markers. In these cases a measure of marker differentiation or divergence becomes an important factor in selecting markers to type. The informativeness metric In, proposed by Rosenberg et al. (2003) is a useful measure of individual divergence per locus and per population comparison that can help guide marker choice ahead of commitments to the necessary genotyping.

The best markers for the inference of ancestry membership proportions are those that efficiently distinguish different populations, i.e., markers showing different alleles at very high frequency in distinct parental populations. Since fixed variation, private to one population, is very rare (Pfaff et al., 2004; Lao et al., 2006) marker selection must always be broadened to loci with maximized allele frequency differences between ancestral populations - these are usually termed Ancestry Informative Markers or AIMs (Yang et al., 2005; Enoch et al., 2006; Salas et al., 2006). Autosomal SNPs are increasingly favored for human population analysis because, in addition to their widespread genomic distribution and ease of genotyping in very dense marker arrays, they are independent of admixture sex bias that routinely affects the distribution of variation of the Y chromosome or mitochondrial DNA. Segregating autosomal markers allow a more thorough measure of admixture in an individual contributed by all of their ancestors rather than just those of single uni-parental lineages (Lao et al., 2006; Phillips et al., 2007; Halder et al., 2008; Royal et al., 2010).

\section{REFERENCE SAMPLES AND VARIATION DATABASES}

Amongst the objectives of human population genetics is the measurement of population-related parameters (e.g., effective size, degrees of relatedness, effects of local natural selection), the detection of admixture and the reconstruction of past demographic events. Therefore, the proper definition of population structure is a key step in studying the populations of a region. In the case of admixed populations it is particularly important to define the original contributing populations by characterizing reference populations and databases of human variation forming the primary data sources for such studies.

A good starting point for collating human SNP variation data from the most extensive catalogs and for standard reference populations is SPSmart [http://spsmart.cesga.es, (Amigo et al., 2008)]. SPSmart has the advantage of being inclusive of all current SNP 


\section{$K=4$}

No Admixture

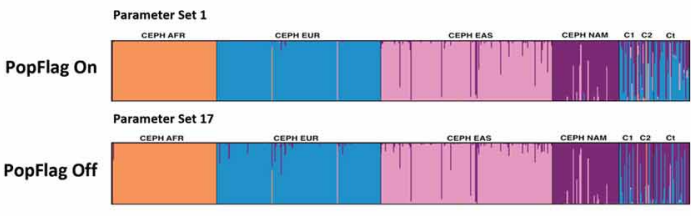

No Admixture LOCPRIOR

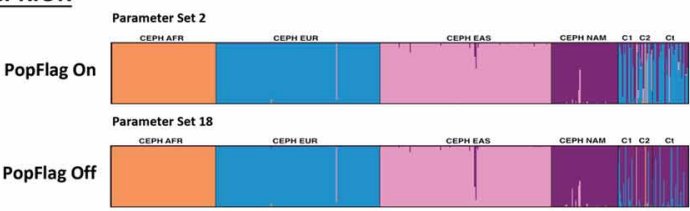

Admixture

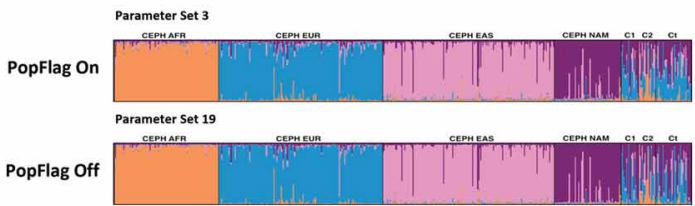

\section{Admixture LOCPRIOR}

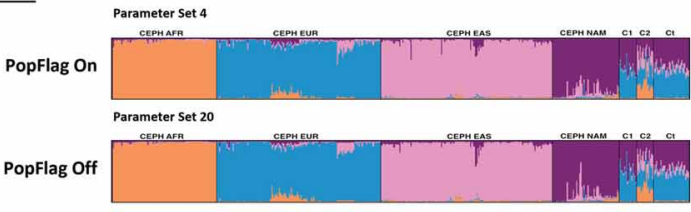

Linkage

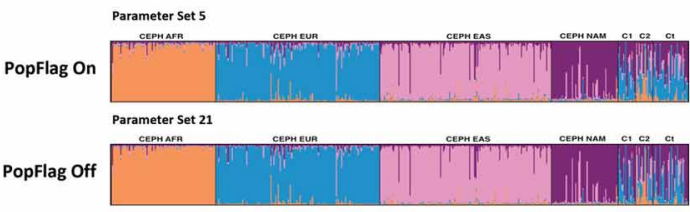

POPINFO No Admixture

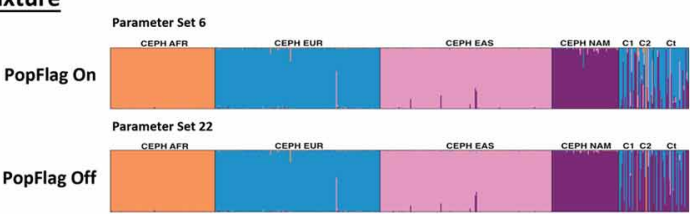

POPINFO Admixture

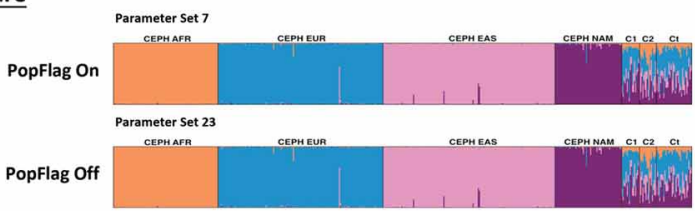

\section{POPINFO Linkage}

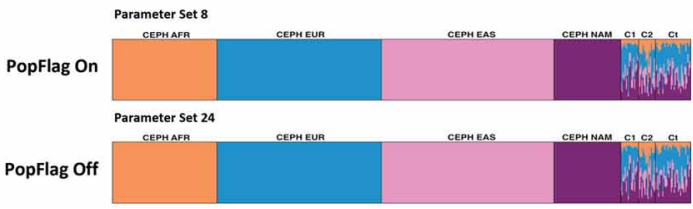

Allele Frequencies Independent
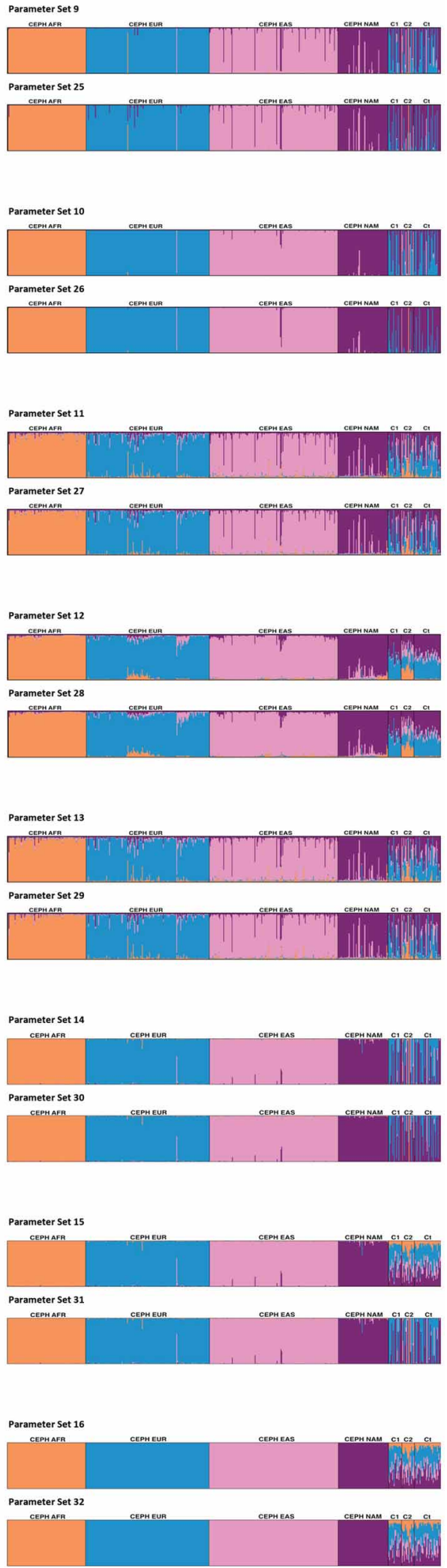

FIGURE 1 | Continued 
FIGURE 1 | STRUCTURE bar plots representing $K=\mathbf{4}$ for the principal analysis parameter combinations available to the user. These graphics were obtained with distruct and using CLUMPP to align the three replicates for $K=4$ (all runs were performed with 100,000 burnin period and 100,000 MCMC repeats after burnin). The exception was the POPINFO parameter sets for which direct STRUCTURE bar plot outputs were used. Human genetic data comprised genotypes listed in Table S1 consisting of 100 Africans: CEPH AFR, 158 Europeans: CEPH EUR, 165 East Asians: CEPH EAS, and 64 Native Americans: CEPH NAM from the
HGDP-CEPH human diversity panel. An artificial case-control group was created using HapMap Mexican and Puerto Rican samples giving a total 67 sample divided into Cases 1 (C1), Cases 2 (C2), and Controls (Ct). Markers were: 9 AIM-SNPs (two triallelic), 3 phenotype associated SNPs and 5 AIM-SNPs on the X-chromosome. The phenotype and the X-SNPs are linked forming two distinct linkage disequilibrium groups-their genetic distance was used to define linkage disequilibrium groups. Each parameter setting and the results obtained are described in detail in

Supplementary Material 1. databases, specifically: 1000 Genomes, HapMap, Perlegen, and Universities of the Stanford and Michigan CEPH-HGDP repositories. Additionally SPSmart allows the collection of genotype data from a large number of markers at a time and their direct transfer into population analysis programs of choice, including STRUCTURE (although some data re-arrangement is necessary to create the input file).

The HGDP-CEPH is frequently used as a panel of population reference samples and $\mathrm{CEPH}$ panel samples from the same predefined population analysed with STRUCTURE nearly always share similar membership coefficients in inferred clusters (Pritchard et al., 2000a; Rosenberg et al., 2002; Jobling et al., 2004; Enoch et al., 2006; Abdulla et al., 2009). Royal et al. (2010) noted that there are some limitations to the accuracy of ancestry inference within and among regions that may be the result of the incomplete sampling by the CEPH-HGDP of total human genetic diversity (Ashg, 2008). The first study using STRUCTURE was performed in 2002 by Rosenberg et al. using 377 microsatellites to infer human population structure in the HGDP-CEPH worldwide population sample. This study concluded that global populations could be grouped into six major discrete ancestral groups matching well with continental distributions. Subsequent studies confirmed that when individuals are grouped on the basis of genetic similarity, group membership corresponds closely to predefined regional or population groups or to collections of geographically and linguistically similar populations (Allocco et al., 2007; Li et al., 2008). In particular, the study of Li et al. (2008) using FRAPPE, a very similar alternative population clustering method to STRUCTURE, divided the HGDP-CEPH into seven major population groups. Furthermore, such studies indicated it was also possible to infer the ancestry of individuals from recently admixed populations in the context of the contributions of putative parental populations (Yang et al., 2005). Mixed ancestries inferred from genetic data can often be interpreted as arising from recent admixture among multiple founder populations. However, it can also be the result of a shared ancestry before the divergence of the two populations with a lack of subsequent gene flow between them (Li et al., 2008).

\section{CASE-CONTROL ASSOCIATION STUDIES}

Case-control association studies (CCAS) provide powerful strategies to identify loci contributing to complex disease. The simplest approach genotypes markers in samples of cases and unrelated controls then tests these for allele frequency differences at each marker-association of genomic regions indicates the loci they contain are possibly linked to disease susceptibility or the presence or absence of particular phenotypes (Pritchard and Donnelly, 2001). However, presence of population structure between case and control groups can produce confounding effects where high false positive rates from allele frequency differences between subpopulations mimics associations with the studied disease. Cryptic relatedness from undetected kinship amongst study subjects is a further confounding effect with potential to exaggerate false positive rates (Voight and Pritchard, 2005; Astle and Balding, 2009). Its effects can be negligible for well-designed studies in outbred populations but cryptic relatedness is more significant in effect for small and isolated populations, when extensive inbreeding occurs or with bias in sample collection toward relatives (Voight and Pritchard, 2005; Astle and Balding, 2009).

Two main approaches are favored to overcome effects of hidden or cryptic structure when it exists between case and control groups: genomic control (GC) and structured association (SA). Pritchard and Donnelly (2001) reviewed GC methods using chisquare tests to detect population stratification through estimation of increase in the test statistic null distribution compared to those of unlinked markers typed in the same group. Using the adjusted distribution gives corrected $p$-values at any given locus. SA approaches use additional genotype information from unlinked markers to estimate the number of subpopulations and each individual's assignment to these subpopulations. This information can then be used to construct a test for association (Pritchard and Donnelly, 2001). SA methods perform well but are computationally demanding and very reliant on estimating the correct number of subpopulations. The recent development of faster model-based methods such as that implemented by ADMIXTURE makes the application of SA methods more feasible (Price et al., 2010). In comparison, GC methods while faster and more straightforward can lack power in certain scenarios, for example, when the markers used are not informative for population comparisons or in the case of cryptic relatedness (Price et al., 2010).

Procedures based on logistic regression that are flexible, computationally fast, and easy to implement, provide protection against the effects of cryptic substructure, even though not explicitly modeling the population structure (Setakis et al., 2006). However, if there is enough information for reliable estimation of sub-population data, the power and flexibility of SA approaches, facilitated by dedicated software such as STRAT, makes them preferable to GC methods (Pritchard and Donnelly, 2001; Price et al., 2008). In fact, analyses performed by Karkkainen and Sillanpaa (2012) demonstrated that in most cases Bayesian multilocus association approaches improve the accuracy of association studies and avoid false positive results. STRAT is applicable to association mapping, enabling valid case-control studies even 
in the presence of population structure. This method was first described by Pritchard et al. (2000b). The application of STRAT improves association studies as it takes into account the confounding effects of population stratification using selected panels of AIMs. Figure 2 summarizes typical STRAT analyses with stratification between case and control samples vs. stratification absent. STRAT chi-square $p$-values are listed for 17 markers directly comparable to STRUCTURE bar plots above. This worked example is discussed in detail in Supplementary Material 1, section 5. It should be noted that as well as common SNPs showing stratification, loci with low frequency $(1-5 \%)$ or rare $(\leq 1 \%)$ minor alleles can inflate the false positive rate significantly (Babron et al., 2012; Mathieson and McVean, 2012) while showing different patterns of stratification from the same population comparisons. The implication of the Babron et al. study is that population analyses may be required for each class of SNP variation when high density SNP data is used for the CCAS.

Several studies have applied STRAT-based stratification control, when there is doubt about the validity of the associations found (i.e., not spurious associations from population stratification) or when it is known that two or more populations are admixed (Han et al., 2008; Tian et al., 2008). The accuracy of inferences improves with sample size, number of loci, and degree of divergence between populations (Pritchard et al., 2000a). An instructive study by Campbell et al. (2005) analyzed the efficacy of stratification control by constructing a case-control group based on adult height then measuring stratification with 111 random SNPs and 67 AIMs. Both SNP sets failed to detect stratification between case and controls but one SNP in LCT (rs4988235: LCT-13910C $\rightarrow$ T) with a frequency difference between north and south Europe co-incidental with average height difference across this geographic distance was strongly associated. Re-matching case and control subjects into equivalent numbers of north and south Europeans in each loses the spurious association. Price et al. (2008) developed an AIM panel capable of distinguishing northwest and south-east European ancestry that applied to Campbell's study efficiently detected stratification. These studies demonstrate the importance of careful marker selection, particularly analysing closely related populations.

Finally a further class of model to detect and correct for stratification is the mixed model-this approach should perform better when confounding effects are present since it simultaneously addresses population's stratification, family structure, and cryptic relatedness (Price et al., 2010). Mixed model methods are computationally challenging but the optimization implemented has enabled large datasets typical of GWAS studies to be readily analysed (Zhang et al., 2010).

\section{OTHER APPLICATIONS: FORENSIC ANALYSIS}

Forensic DNA analysis is a powerful tool in near-universal use as a core part of police investigations. DNA profiling is particularly informative used in conjunction with DNA databases of
A

\section{Stratification absent}

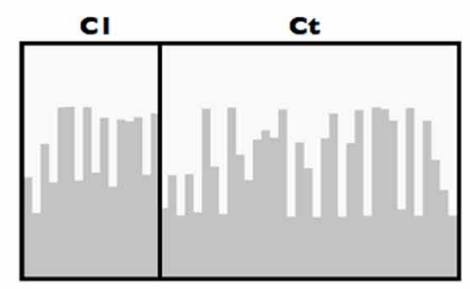

B

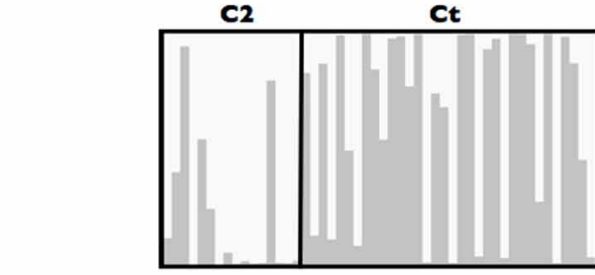

1: chisq $=5.583 \quad 1 \mathrm{df}$;

2: $\operatorname{chisq}=21.140 \quad 1 d f$;

3: $\operatorname{chisq}=0.949 \quad 1 \mathrm{df}$;

4: $\quad$ chisq $=0.563 \quad 1 d f$;

5: $\quad$ chisq $=0.046 \quad 1 d f$;

6: $\operatorname{chisq}=1.968 \quad 1 \mathrm{df}$;

7: chisq=9.631 1df;

8: chisq $=0.461 \quad 1 d f$;

9: chisq=16.185 1df;

: $\quad$ chisq $=0.003 \quad 1 d f$;

11: chisq $=0.0851 \mathrm{df}$;

2: chisq $=0.506 \quad 1 \mathrm{df}$;

3: $\operatorname{chisq}=10.725 \quad 1 d f$;

4: chisq=14.299 1df;

5: $\operatorname{chisq}=0.158 \quad 1 \mathrm{df}$;

: chisq $=8.316 \quad 1 \mathrm{df}$;

chisq=5.823 $1 \mathrm{df}$;
$\mathrm{TS}=4.71$,

$\mathrm{TS}=9.12$,

$\mathrm{TS}=0.50$,

$\mathrm{TS}=2.15$,

$\mathrm{TS}=0.53$,

$\mathrm{TS}=1.08$,

$\mathrm{TS}=5.97$,

$\mathrm{TS}=0.27$,

$\mathrm{TS}=4.83$,

$\mathrm{TS}=1.80$,

$\mathrm{TS}=0.12$,

$\mathrm{TS}=3.39$,

$\mathrm{TS}=0.08$,

$\mathrm{TS}=1.90$,

$\mathrm{TS}=0.78$,

$\mathrm{TS}=0.37$,

$\mathrm{TS}=0.16$,

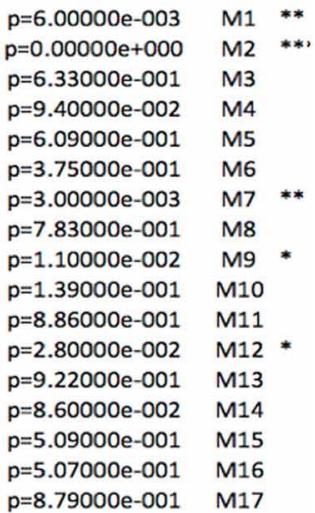

$\mathrm{p}=8.79000 \mathrm{e}-001 \quad \mathrm{M} 17$

Summary of distribution of p-values

$\begin{array}{cccccccccc}0.00--0.05: & 0.176 & 0.059 & 0.059 & 0.000 & 0.000 & 0.2941 & 0.2941 & 0.2441\end{array}$
Summary of distribution of p-values
$\begin{array}{llllllllll}0.00--0.05: & 0.000 & 0.000 & 0.000 & 0.000 & 0.063 & 0.0625 & 0.0625 & 0.0125\end{array}$

FIGURE 2 | Example case-control sample analyses comparing scenarios with the presence or absence of stratification.

STRUCTURE bar plots and STRAT table results are shown. (A) Case 1
(C1) are compared to the Control (Ct) samples. (B) Case 2 (C2) are compared to the Control (Ct) samples. Details of these analyses are described in Supplementary Material 1 
offender profiles. Although situations occur with no database match, so any additional information that can be obtained from the DNA becomes valuable (Lowe et al., 2001). Information indicating probable ancestry of an unknown offender can help direct investigations towards a smaller suspect group (Lowe et al., 2001; Phillips et al., 2011). Likewise interest is growing in the prediction of externally visible characteristics such as eye color (Ruiz et al., 2013; Walsh et al., 2013). Currently microsatellite short tandem repeat (STR) typing is the standard approach and still the method of choice, providing extremely high discrimination power for most problems of human identification (Chakraborty et al., 1999; Butler, 2005). Several studies have concluded STR profiles can enable ancestry inference (Bowcock et al., 1994; Graydon et al., 2009; Londin et al., 2010; Phillips et al., 2011), but only with sufficient reliability when other AIMs such as SNPs are included (Phillips et al., 2011). One example of the benefits from adding specialized marker sets to enhance ancestry analysis in forensic casework is the 11-M Madrid bomb attack investigations where results of a 34-SNP ancestry test were analysed with STRUCTURE to infer the probable ancestry of suspects (Phillips et al., 2009). One disadvantage of STRUCTURE highlighted by this study is the difficulty of analyzing single genotype profiles. An alternative online classifier termed Snipper (Phillips et al., 2007), analyzes single profiles with a near identical Bayesian algorithm and gives likelihoods of membership to ancestry groups inferred from user-defined training sets acting as the reference material.

\section{ALTERNATIVE POPULATION ANALYSIS APPROACHES}

Several population analysis programs provide alternatives to STRUCTURE and are applicable to most of the analyses outlined above. Comparisons of many of these programs are presented in Table 1. A more comprehensive review by Liu et al. (2013) assesses regularly chosen alternative software and methods for population analysis. Such programs are usually designed for a specific population analysis application so they lack the major advantage of STRUCTURE in offering the flexibility to adapt to varying analysis demands. Although many options are available for analysis of population data and inference of genetic ancestry there is no one program applicable to all situations or data types. However, STRUCTURE can readily handle different markers and their characteristics (SNPs, STRs, linked markers, and loci with dominance). Provision of different ancestry and allele frequency models allows the user to adapt different analyses in a straightforward way with a unified approach based on STRUCTURE alone or combined with supporting programs. The adaptability of STRUCTURE is underlined by its widespread application to population genetics studies in general or specifically to forensic analysis and stratification adjustment of CCAS.

\section{THE STEP-BY-STEP GUIDE TO STRUCTURE ANALYSIS}

The user guide to STRUCTURE in Supplementary Material 1, comprises a step-by-step outline and covers the fundamentals of creating an input file and project, the available analysis models, the definition of parameter sets, and how to run a simulation. The guide runs through an example where each of the analysis models and principal parameters of the four software tools are explored. The genotypic data used in this example is available in Table S1. We present suggestions for the analysis and graphical display of the results and optimum estimation of the number of populations detected in a dataset. Additionally, we describe how to handle the parameters included in CLUMPP, distruct and STRAT. CLUMPP allows the alignment of different replicates of STRUCTURE analysis results from a given number of assumed populations, helping to deal with the commonly encountered problem of multimodality. Clustering algorithms such as the one implemented in STRUCTURE can include stochastic simulations during the inferences. This creates a results space composed of different membership coefficients, each one with an associated probability. It is possible that, when analysing the same data set with identical conditions, different final results are obtained. Differences between replicated analysis runs can be of two types: label change or genuine multimodality. Label change occurs when different replicates create the same membership coefficient estimates but the labels of each group are distinct in each permutation, that is, each cluster does not represent the same predefined population in all runs. It is also possible that the replicates create distinct but likely results that are not equivalent between permutations - genuine multimodalitythat is, clusters that represent a particular predefined population have different ancestry membership proportions in each run. This can be the result of difficulties in the search for the possible membership coefficients space or of true biological factors (Jakobsson and Rosenberg, 2007).

Independently of genuine differences between a series of replicated analyses, a method is needed to deal with the replicate results obtained from multiple runs analysing a single dataset. CLUMPP software provides three algorithms that identify the best alignment to the replicate results of the cluster analysis. CLUMPP reviews the membership coefficient matrices finding those replicates with the best correspondence. Both STRUCTURE and CLUMPP give similar output files to the extent that CLUMPP results can be directly used in standard STRUCTURE graphic enhancement software such as distruct described below.

An informative way to visualize STRUCTURE results is to show each individual as a column segmented into $K$ colors representing the estimated membership coefficients. Distruct software offers a wide range of options to create images based on the principle of segmented columns and provides visually appealing graphical summaries of the population structure detected in the data. Distruct is required by CLUMPP but usefully provides an alternative graphical display to the standard STRUCTURE bar plots, offering a wider variety of options to create images for much of the STRUCTURE output.

Finally, STRAT can be used in association studies, enabling the validation of case-control association statistics even in the presence of population structure that can compound the associations suggested by the data.

We focus this work on the 2.3.3 front-end version of STRUCTURE but a new version (V2.3.4) was recently released to fix some minor bugs. It is worth noting that the recently released StrAuto v0.3.1 Python-based software enables an 


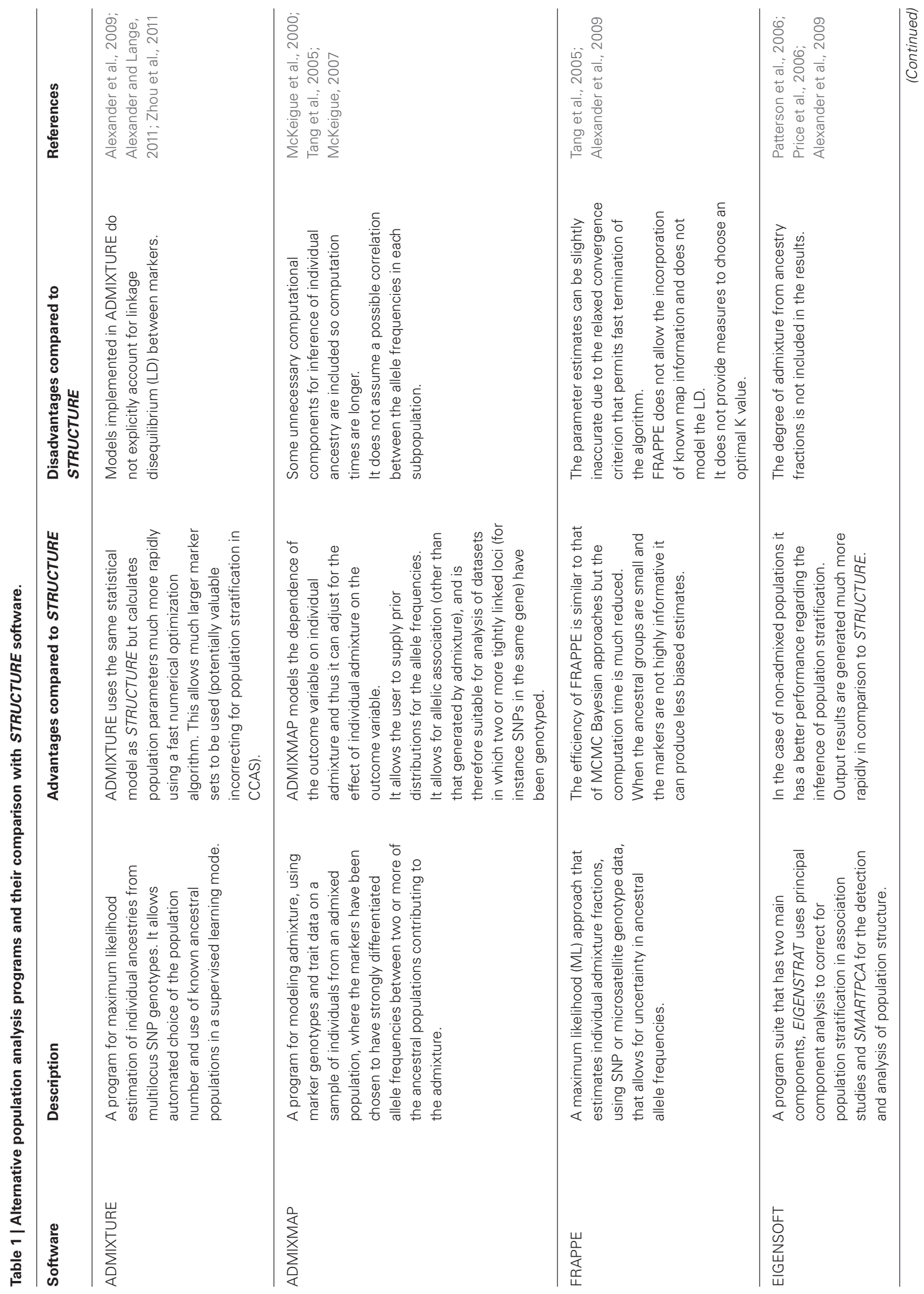



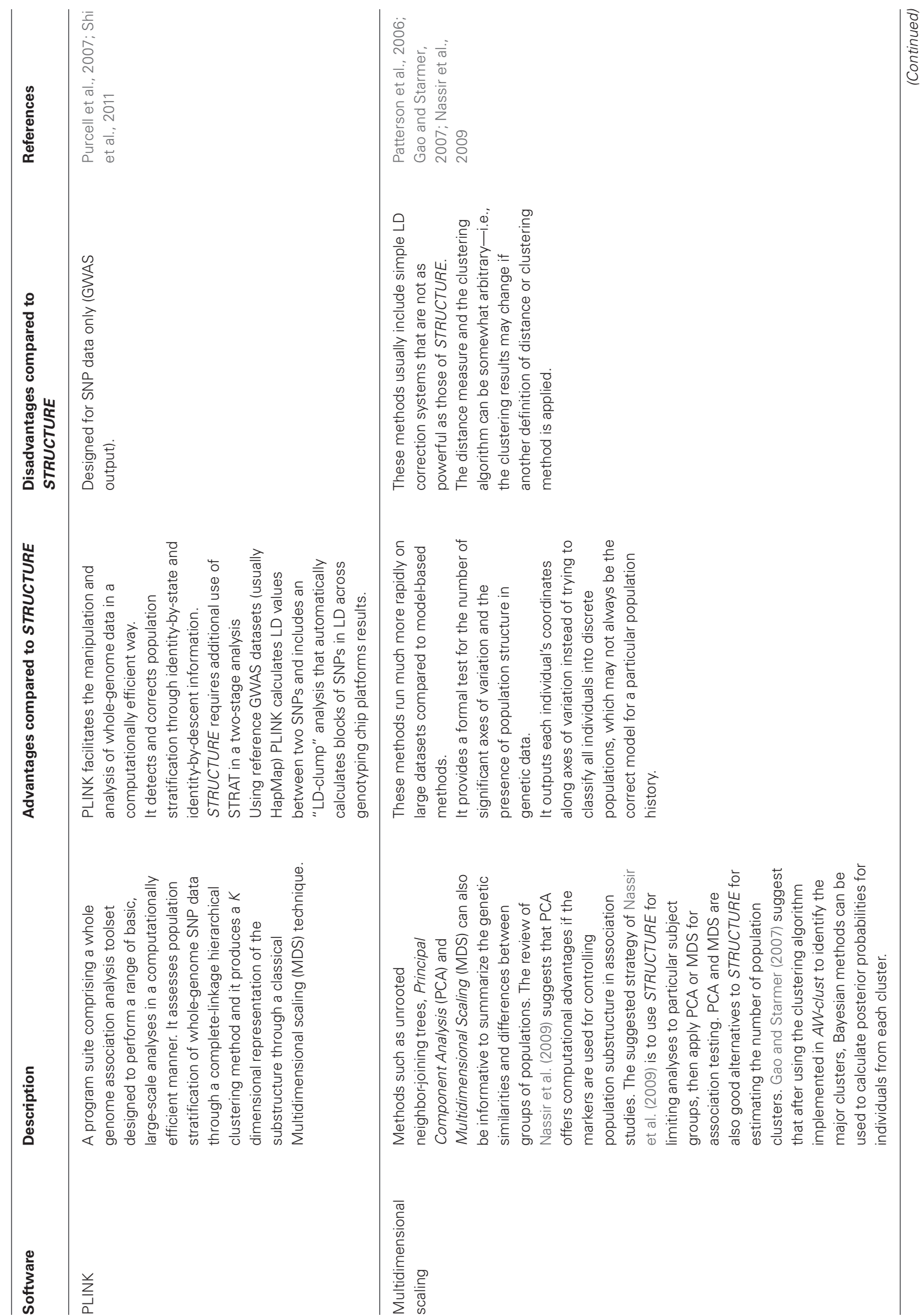


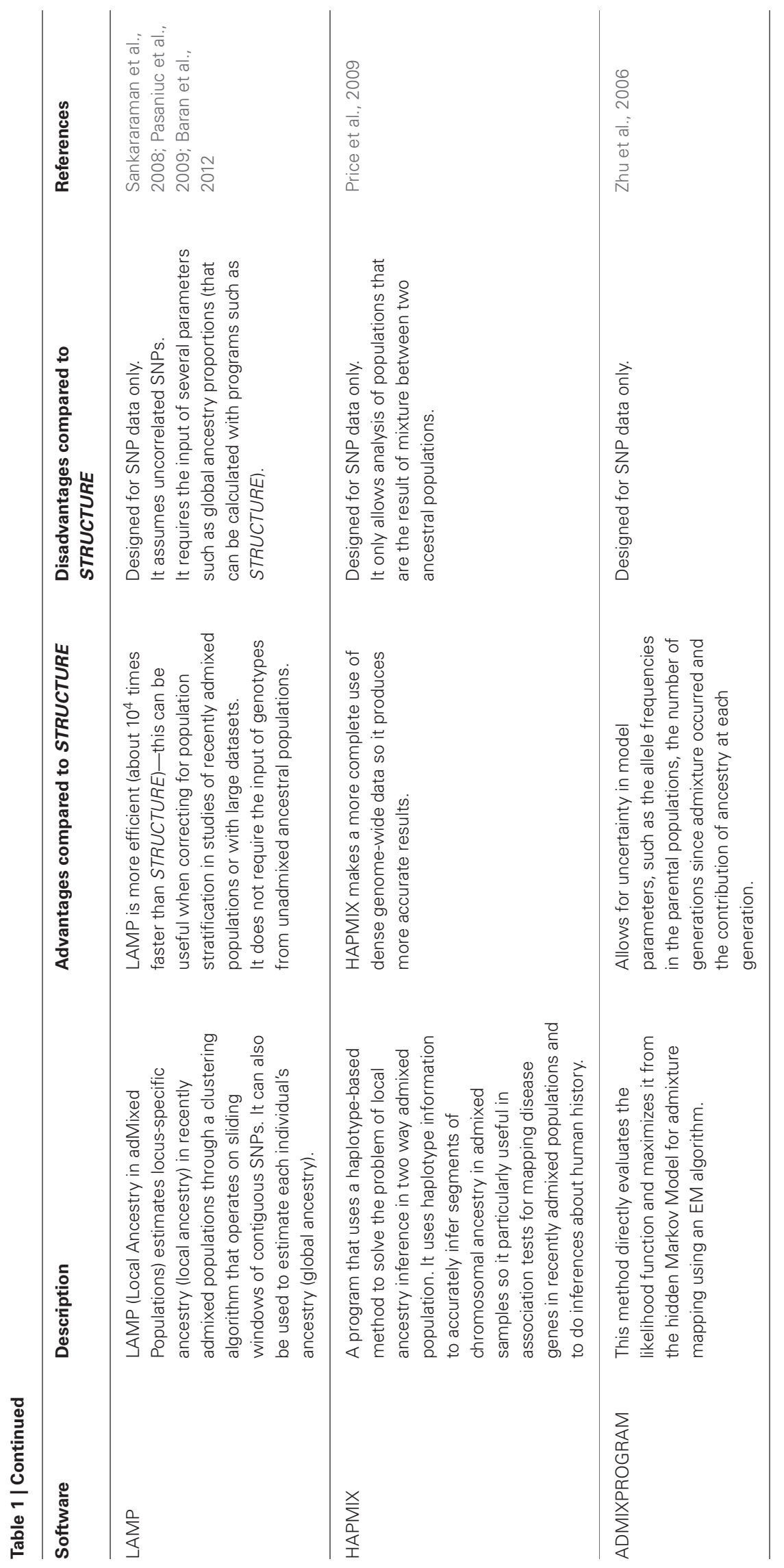


automated approach, albeit from the command line of Mac or Linux based computers (Chhatre, 2012).

\section{CONCLUDING REMARKS}

This article presents an updated review of the ubiquitous STRUCTURE population analysis software widely applied to a range of population genetics problems. We give recommendations that can guide decisions when analyzing population structure for population genetics and association studies. The review and guide focuses on STRUCTURE and the supporting software of CLUMPP, distruct and STRAT. The use of a Bayesian method offers several advantages, especially assigning admixed individuals to population clusters, since it is possible to use prior information to assist the calculation of ancestry proportions for these individuals. Therefore, information on data, the markers applied and the type of analysis desired is relevant before the selection of the analysis parameters.

A simulated example file was thoroughly analyzed and our concluding remark is that there is no one standard analysis parameter in STRUCTURE - the data and the study objectives will influence the choice of the most appropriate parameterand precaution should be used to avoid overestimating the actual population structure present in complex data.

\section{REFERENCES}

Abdulla, M. A., Ahmed, I., Assawamakin, A., Bhak, J., Brahmachari, S. K., Calacal, G. C., et al. (2009). Mapping human genetic diversity in Asia. Science 326, 1541-1545. doi: 10.1126/ science. 1177074

Alexander, D. H., and Lange, K. (2011). Enhancements to the ADMIXTURE algorithm for individual ancestry estimation. BMC Bioinformatics 12:246. doi: 10.1186/1471-210512-246

Alexander, D. H., Novembre, J., and Lange, K. (2009). Fast model-based estimation of ancestry in unrelated individuals. Genome Res. 19, 1655-1664. doi: 10.1101/gr. 094052.109

Allocco, D. J., Song, Q., Gibbons, G. H., Ramoni, M. F., and Kohane, I. S. (2007). Geography and genography: prediction of continental origin using randomly selected single nucleotide polymorphisms. BMC Genomics 8:68. doi: 10.1186/14712164-8-68

Amigo, J., Salas, A., Phillips, C., and Carracedo, A. (2008). SPSmart: adapting population based SNP genotype databases for fast and comprehensive web access. BMC Bioinformatics 9:428. doi: 10.1186/1471-2105-9-428

Ashg. (2008). The American Society Of Human Genetics: Ancestry Testing Statement [Online]. Available online at: http://www.ashg.org/pdf/ ASHGAncestryTestingStatement_ FINAL.pdf. (Accessed November 13, 2008).

Astle, W., and Balding, D. J. (2009). Population structure and cryptic relatedness in genetic association studies. Statist. Sci. 24, 451-471. doi: 10.1214/09-STS307

Babron, M. C., De Tayrac, M., Rutledge, D. N., Zeggini, E., and Genin, E. (2012). Rare and low frequency variant stratification in the UK population: description and impact on association tests. PLoS ONE 7:e46519. doi: 10.1371/journal. pone.0046519

Baran, Y., Pasaniuc, B., Sankararaman, S., Torgerson, D. G., Gignoux, C., Eng, C., et al. (2012). Fast and accurate inference of local ancestry in Latino populations. Bioinformatics formatics/bts144

Bowcock, A. M., Ruiz-Linares, A., Tomfohrde, J., Minch, E., Kidd, J. R., and Cavalli-Sforza, L. L. (1994). High resolution of human evolutionary trees with polymorphic microsatellites. Nature 368, 455-457. doi: 10.1038/ $368455 \mathrm{a} 0$

Butler, J. M. (2005). Forensic DNA Typing. Burlington, VT: London Elsevier Academic Press.

Campbell, C. D., Ogburn, E. L., Lunetta, K. L., Lyon, H. N., Freedman, M. L., Groop, L. C., 28, 1359-1367. doi: 10.1093/bioin-

\section{ACKNOWLEDGMENTS}

Liliana Porras-Hurtado is supported by funding from Colciencias Colombia. Yarimar Ruiz is supported by funding from the Fundación Gran Mariscal de Ayacucho (FUNDAYACUCHO) E228-585-2007-1. Carla Santos is supported by funding awarded by the Portuguese Foundation for Science and Technology (FCT) and co-financed by the European Social Fund [Human Potential Thematic Operational Programme (SFRH/BD/75627/2010)]. Maria V. Lareu is supported by funding from Xunta de Galicia INCITE 09208163PR. The authors wish to thank Antonio Salas of the Forensic Genetics Unit, University of Santiago de Compostela and Jeremy Austin of the Australian Centre for Ancient DNA, University of Adelaide, for helpful discussions and guidance in the preparation of the manuscript.

\section{SUPPLEMENTARY MATERIAL}

The Supplementary Material for this article can be found online at: http://www.frontiersin.org/Statistical_Genetics_and_ Methodology/10.3389/fgene.2013.00098/abstract

\section{Supplementary Material 1 | A step-by-step guide to STRUCTURE and accompanying data processing programs.}

Table S1 | Excel file of genotypes and STRUCTURE input data used in the example analyses of Supplementary Material 1.

et al. (2005). Demonstrating stratification in a European American population. Nat. Genet. 37, 868-872. doi: 10.1038/ng1607

Cann, H. M., De Toma, C., Cazes, L., Legrand, M.-F., Morel, V., Piouffre, L., et al. (2002). A human genome diversity cell line panel. Science 296, 261-262. doi: 10.1126/science.296.5566.261b

Carracedo, A., Butler, J. M., Gusmao, L., Linacre, A., Parson, W., Roewer, L., et al. (2013). New guidelines for the publication of genetic population data. Forensic Sci. Int. Genet. 7, 217-220. doi: 10.1016/j.fsigen.2013.01.001

Chakraborty, R., Stivers, D. N., Su, B., Yixi, Z., and Bruce, B. (1999). The utility of short tandem repeat loci beyond human identification: implications for development of new DNA typing systems. Electrophoresis 20, 1682-1696. doi: $\quad 10.1002 /($ SICI) $1522-2683$ (19990101)20:8<1682::AID-ELPS $1682>3.0 . C O ; 2-Z$

Chhatre, V. E. (2012). StrAuto ver0.3.1: A python Program - Automation of Structure Analysis. Available online at: http://www.crypticline age.net/pages/software.html.

Corander, J., and Marttinen, P. (2006). Bayesian identification of admixture events using multilocus molecular markers. Mol. Ecol. 15, 2833-2843. doi: 10.1111/j.1365-294X.2006.02994.x
Corander, J., Waldmann, P., and Sillanpaa, M. J. (2003). Bayesian analysis of genetic differentiation between populations. Genetics 163, 367-374.

Enoch, M. A., Shen, P. H., Xu, K., Hodgkinson, C., and Goldman, D. (2006). Using ancestry-informative markers to define populations and detect population stratification. J. Psychopharmacol. 20, 19-26. doi: 10.1177/1359786806066041

Falush, D., Stephens, M., and Pritchard, J. K. (2003). Inference of population structure using multilocus genotype data: linked loci and correlated allele frequencies. Genetics 164, 1567-1587. doi: 10.3410/f.1015548.197423

Falush, D., Stephens, M., and Pritchard, J. K. (2007). Inference of population structure using multilocus genotype data: dominant markers and null alleles. Mol. Ecol. Notes 7, 574-578. doi: 10.1111/j.14718286.2007.01758.x

Gao, X., and Starmer, J. (2007). Human population structure detection via multilocus genotype clustering. BMC Genet. 8:34. doi: 10.1186/1471-2156-8-34

Graydon, M., Cholette, F., and Ng, L. K. (2009). Inferring ethnicity using 15 autosomal STR loci-comparisons among populations of similar and distinctly different physical traits. Forensic Sci. Int. Genet. 3, 251-254. doi: 10.1016/j.fsigen.2009.03.002 
Halder, I., Shriver, M., Thomas, M., Fernandez, J. R., and Frudakis, T. (2008). A panel of ancestry informative markers for estimating individual biogeographical ancestry and admixture from four continents: utility and applications. Hum. Mutat. 29, 648-658. doi: 10.1002/humu.20695

Han, S., Guthridge, J. M., Harley, I. T. W., Sestak, A. L., Kim-Howard, X., Kaufman, K. M., et al. (2008). Osteopontin and systemic lupus erythematosus association: a probable gene-gender interaction. PLoS ONE 3:e0001757. doi: 10.1371/journal.pone. 0001757

Hubisz, M. J., Falush, D., Stephens, M., and Pritchard, J. K. (2009). Inferring weak population structure with the assistance of sample group information. Mol. Ecol. Res. 9, 1322-1332. doi: 10.1111/j.17550998.2009.02591.x

Jakobsson, M., and Rosenberg, N. A. (2007). CLUMPP: a cluster matching and permutation program for dealing with label switching and multimodality in analysis of population structure. Bioinformatics 23, 1801-1806. doi: 10.1093/bioinformatics/btm233

Jobling, M. A., Hurles, M., and Tyler-Smith, C. (2004). Human Evolutionary Genetics: Origins, Peoples and Disease. New York, NY: Garland Science - Taylor and Francis Group.

Kalinowski, S. T. (2011). The computer program STRUCTURE does not reliably identify the main genetic clusters within species: simulations and implications for human population structure. Heredity 106, 625-632. doi: 10.1038/hdy. 2010.95

Karkkainen, H. P., and Sillanpaa, M. J. (2012). Robustness of Bayesian multilocus association models to cryptic relatedness. Ann. Hum. Genet. 76, 510-523. doi: 10.1111/j.1469-1809.2012.00729.x

Lao, O., Van Duijn, K., Kersbergen, P., De Knijff, P., and Kayser, M. (2006). Proportioning wholegenome single-nucleotide-polymorphism diversity for the identification of geographic population structure and genetic ancestry. $A m$. J. Hum. Genet. 78, 680-690. doi: $10.1086 / 501531$

Li, J. Z., Absher, D. M., Tang, H., Southwick, A. M., Casto, A. M., Ramachandran, S., et al. (2008). Worldwide human relationships inferred from genome-wide patterns of variation. Science 319, 1100-1104. doi: 10.1126/science. 1153717
Liu, Y., Nyunoya, T., Leng, S., Belinsky, S. A., Tesfaigzi, Y., and Bruse, S. (2013). Softwares and methods for estimating genetic ancestry in human populations. Hum. Genomics 7:1. doi: 10.1186/1479-7364-7-1

Londin, E. R., Keller, M. A., Maista, C., Smith, G., Mamounas, L. A., Zhang, R., et al. (2010). CoAIMs: a costeffective panel of ancestry informative markers for determining continental origins. PLoS ONE 5:e13443. doi: 10.1371/journal.pone.0013443

Lowe, A. L., Urquhart, A., Foreman, L. A., and Evett, I. W. (2001). Inferring ethnic origin by means of an STR profile. Forensic Sci. Int. 119, 17-22. doi: 10.1016/S0379-0738 (00)00387-X

Mathieson, I., and McVean, G. (2012). Differential confounding of rare and common variants in spatially structured populations. Nat. Genet. 44, 243-246. doi: 10.1038/ng.1074

McKeigue, P. (2007). ADMIXMAP: A Program to Model Admixture Using Marker Genotype Data [Online]. Available online at: http://home pages.ed.ac.uk/pmckeigu/admixmap/ admixmap_manual.pdf. (Accessed April 25, 2013).

McKeigue, P. M., Carpenter, J. R., Parra, E. J., and Shriver, M. D. (2000). Estimation of admixture and detection of linkage in admixed populations by a Bayesian approach: application to African-American populations. Ann. Hum. Genet. 64, 171-186. doi: 10.1017/S0003480000008022

Nassir, R., Kosoy, R., Tian, C., White, P., Butler, L., Silva, G., et al. (2009). An ancestry informative marker set for determining continental origin: validation and extension using human genome diversity panels. BMC Genet. 10:39. doi: 10.1186/1471-215610-39

Pasaniuc, B., Sankararaman, S., Kimmel, G., and Halperin, E. (2009). Inference of locus-specific ancestry in closely related populations. Bioinformatics 25, i213-i221. doi: 10.1093/bioinformatics/btp 197

Patterson, N., Price, A. L., and Reich, D. (2006). Population structure and eigenanalysis. PLoS Genet. 2:e190. doi: 10.1371/journal.pgen.0020190

Pfaff, C. L., Barnholtz-Sloan, J., Wagner, J. K., and Long, J. C. (2004). Information on ancestry from genetic markers. Genet. Epidemiol. 26, 305-315. doi: 10.1002/gepi.10319

Phillips, C., Fernandez-Formoso, L., Garcia-Magarinos, M., Porras, L., Tvedebrink, T., Amigo, J., et al.
(2011). Analysis of global variability in 15 established and 5 new European Standard Set (ESS) STRs using the CEPH human genome diversity panel. Forensic Sci. Int. Genet. 5, 155-169. doi: 10.1016/j.fsigen.2010.02.003

Phillips, C., Prieto, L., Fondevila, M., Salas, A., Gomez-Tato, A., Alvarez-Dios, J., et al. (2009). Ancestry analysis in the 11-M Madrid bomb attack investigation. PLOS ONE 4:e6583. doi: 10.1371/journal.pone.0006583

Phillips, C., Salas, A., Sanchez, J. J., Fondevila, M., Gomez-Tato, A., Alvarez-Dios, J., et al. (2007). Inferring ancestral origin using a single multiplex assay of ancestryinformative marker SNPs. Forensic Sci. Int. Genet. 1, 273-280. doi: 10.1016/j.fsigen.2007.06.008

Price, A. L., Butler, J., Patterson, N., Capelli, C., Pascali, V. L., Scarnicci, F., et al. (2008). Discerning the ancestry of European Americans in genetic association studies. PLoS Genet. 4:e236. doi: 10.1371/journal.pgen.0030236

Price, A. L., Patterson, N. J., Plenge, R. M., Weinblatt, M. E., Shadick, N. A., and Reich, D. (2006). Principal components analysis corrects for stratification in genome-wide association studies. Nat. Genet. 38, 904-909. doi: 10.1038/ng1847

Price, A. L., Tandon, A., Patterson, N., Barnes, K. C., Rafaels, N., Ruczinski, I., et al. (2009). Sensitive detection of chromosomal segments of distinct ancestry in admixed populations. PLoS Genet. 5:e1000519. doi: 10.1371/journal.pgen.1000519

Price, A. L., Zaitlen, N. A., Reich, D., and Patterson, N. (2010). New approaches to population stratification in genome-wide association studies. Nat. Rev. Genet. 11, 459-463. doi: 10.1038/nrg2813

Pritchard, J. K., and Donnelly, P. (2001). Case-control studies of association in structured or admixed populations. Theor. Popul. Biol. 60, 227-237. doi: 10.1006/tpbi.2001.1543

Pritchard, J. K., Stephens, M., and Donnelly, P. (2000a). Inference of population structure using multilocus genotype data. Genetics 155 , 945-959.

Pritchard, J. K., Stephens, M., Rosenberg, N. A., Donnelly, P. (2000b). Association Mapping in Structured Populations. Am. J. Hum. Genet. 67, 170-181. doi: 10.1086/302959

Purcell, S., Neale, B., Todd-Brown, K., Thomas, L., Ferreira, M. A., Bender, D., et al. (2007). PLINK: a tool set for whole-genome association and population-based linkage analyses. Am. J. Hum. Genet. 81, 559-575. doi: 10.1086/ 519795

Rosenberg, N. A. (2006). Standardized subsets of the HGDP-CEPH human genome diversity cell line panel, accounting for atypical and duplicated samples and pairs of close relatives. Ann. Hum. Genet. 70, 841-847. doi: 10.1111/j.1469-1809. 2006.00285.x

Rosenberg, N. A., Li, L. M., Ward, R., and Pritchard, J. K. (2003). Informativeness of genetic markers for inference of ancestry. Am. J. Hum. Genet. 73, 1402-1422. doi: $10.1086 / 380416$

Rosenberg, N. A., Pritchard, J. K., Weber, J. L., Cann, H. M., Kidd, K. K., Zhivotovsky, L. A., et al (2002). Genetic structure of human populations. Science 298, 2381-2385. doi: 10.1126/science. 1078311

Royal, C. D., Novembre, J., Fullerton, S. M., Goldstein, D. B., Long, J. C., Bamshad, M. J., et al. (2010). Inferring genetic ancestry: opportunities, challenges, and implications. Am. J. Hum. Genet. 86, 661-673. doi: 10.1016/j.ajhg.2010. 03.011

Ruiz, Y., Phillips, C., Gomez-Tato, A., Alvarez-Dios, J., Casares De Cal, M., Cruz, R., et al. (2013). Further development of forensic eye color predictive tests. Forensic Sci. Int. Genet. 7, 28-40. doi: 10.1016/j.fsigen.2012.05.009

Salas, A., Phillips, C., and Carracedo, A. (2006). Ancestry vs physical traits: the search for ancestry informative markers (AIMs). Int. J. Legal Med. 120, 188-189. doi: 10.1007/s00414005-0032-3

Sankararaman, S., Sridhar, S., Kimmel, G., and Halperin, E. (2008). Estimating local ancestry in admixed populations. Am. J. Hum. Genet. 82, 290-303. doi: 10.1016/j.ajhg.2007.09.022

Setakis, E., Stirnadel, H., and Balding, D. J. (2006). Logistic regression protects against population structure in genetic association studies. Genome Res. 16, 290-296. doi: 10.1101/gr.4346306

Shi, H., Medway, C., Brown, K. Kalsheker, N., and Morgan, K. (2011). Using Fisher's method with PLINK 'LD clumped' output to compare SNP effects across Genome-wide Association Study (GWAS) datasets. Int. J. Mol. Epidemiol. Genet. 2, 30-35.

Shi, W., Ayub, Q., Vermeulen, M., Shao, R. G., Zuniga, S., van der 
Gaag, K., et al. (2010). A worldwide survey of human male demographic history based on Y-SNP and Y-STR data from the HGDPCEPH populations. Mol. Biol. Evol. 27, 385-393. doi: 10.1093/molbev/ msp243

Tang, H., Peng, J., Wang, P., and Risch, N. J. (2005). Estimation of individual admixture: analytical and study design considerations. Genet. Epidemiol. 28, 289-301. doi: 10. 1002/gepi.20064

Tian, C., Plenge, R. M., Ransom, M., Lee, A., Villoslada, P., Selmi, C., et al. (2008). Analysis and application of european genetic substructure using $300 \mathrm{~K}$ SNP information. PLoS Genet. 4:e4. doi: 10.1371/journal.pgen.0040004

Voight, B. F., and Pritchard, J. K. (2005). Confounding from cryptic relatedness in case-control association studies. PLoS Genet. 1:e32. doi: 10.1371/journal.pgen. 0010032
Walsh, S., Liu, F., Wollstein, A., Kovatsi, L., Ralf, A., Kosiniak-Kamysz, A., et al. (2013). The HIrisPlex system for simultaneous prediction of hair and eye colour from DNA. Forensic Sci. Int. Genet. 7, 98-115. doi: 10.1016/j.fsigen.2012. 07.005

Waples, R. S., and Gaggiotti, O. (2006). What is a population? An empirical evaluation of some genetic methods for identifying the number of gene pools and their degree of connectivity. Mol. Ecol. 15, 1419-1439. doi: $\quad 10.1111 /$ j.1365-294X.2006. 02890.x

Yang, N., Li, H. Z., Criswell, L. A., Gregersen, P. K., Alarcon-Riquelme, M. E., Kittles, R., et al. (2005). Examination of ancestry and ethnic affiliation using highly informative diallelic DNA markers: application to diverse and admixed populations and implications for clinical epidemiology and forensic medicine. Hum. Genet. 118,
382-392. doi: 10.1007/s00439-0050012-1

Zhang, Z., Ersoz, E., Lai, C.-Q., Todhunter, R. J., Tiwari, H. K. Gore, M. A., et al. (2010). Mixed linear model approach adapted for genome-wide association studies. Nat. Genet. 42, 355-360. doi: 10.1038/ng.546

Zhou, H., Alexander, D. H., and Lange, K. (2011). A quasi-Newton acceleration for high-dimensional optimization algorithms. Stat. Comput. 21, 261-273. doi: 10.1007/s11222009-9166-3

Zhu, X., Zhang, S., Tang, H., and Cooper, R. (2006). A classical likelihood based approach for admixture mapping using EM algorithm. Hum. Genet. 120, 431-445. doi: 10.1007/s00439-006-0224-z

Conflict of Interest Statement: The authors declare that the research was conducted in the absence of any commercial or financial relationships that could be construed as a potential conflict of interest.

Received: 01 March 2013; accepted: 14 May 2013; published online: 29 May 2013.

Citation: Porras-Hurtado L, Ruiz Y, Santos C, Phillips C, Carracedo Á and Lareu MV (2013) An overview of STRUCTURE: applications, parameter settings, and supporting software. Front. Genet. 4:98. doi: 10.3389/fgene. 2013.00098

This article was submitted to Frontiers in Statistical Genetics and Methodology, a specialty of Frontiers in Genetics.

Copyright (c) 2013 Porras-Hurtado, Ruiz, Santos, Phillips, Carracedo and Lareu. This is an open-access article distributed under the terms of the Creative Commons Attribution License, which permits use, distribution and reproduction in other forums, provided the original authors and source are credited and subject to any copyright notices concerning any third-party graphics etc. 